\title{
Poincaré series and zeta function for an irreducible plane curve singularity
}

\author{
Jan Stevens
}

\begin{abstract}
The Poincaré series of an irreducible plane curve singularity equals the $\zeta$-function of its monodromy, by a result of Campillo, Delgado and GuseinZade. We derive this fact from a formula of Ebeling and Gusein-Zade relating the Poincaré series of a quasi-homogeneous complete intersection singularity to the Saito dual of a product of $\zeta$-functions.
\end{abstract}

Several cases are known where the $\zeta$-function of the monodromy of an isolated hypersurface singularity is related to the Poincaré series of its coordinate ring. The first instance of this phenomenon was observed by Campillo, Delgado and Gusein-Zade [CDG]: for an irreducible plane curve singularity the $\zeta$-function of the monodromy equals its Poincaré series. The proof is by comparing explicit formulas. All attempts have failed at a more direct proof. But maybe this example is misleading. The results of Ebeling and Gusein-Zade [Eb, EGZ] suggest a more indirect connection. For quasi-homogeneous complete intersection singularities they show that the Poincaré series, corrected by an orbit invariant, is related to the Saito dual of a product of $\zeta$-functions. The precise formulation will be given below.

The object of this note is to derive the original result of [CDG] from the formula of [EGZ]. The question remains to give a direct proof of their formula and to explain the meaning of the Saito dual. The key observation, stressed by Teissier in [Zar], is that an irreducible plane curve singularity is a (in some sense equisingular) deformation of a monomial curve with the same semigroup, which is moreover a complete intersection. What is needed is a topological argument, which connects the $\zeta$-function of the plane curve singularity with the product of $\zeta$-functions occurring in the formula of [EGZ].

In the first section we give the definition of the monodromy $\zeta$-function and motivate our conventions by recalling the analogy with number theory. Then we state the formula of Ebeling and Gusein-Zade. In the final section we derive the main result. 


\section{The zeta function of the monodromy}

There is some confusion in the literature about the definition of the $\zeta$-function for singularities. We follow Milnor's book [Mi2]. The definition is based on an analogy with number theory. For a number field $K$ the Dedekind $\zeta$-function is

$$
\zeta_{K}(s)=\prod_{\mathfrak{p}} \frac{1}{1-\frac{1}{\mathcal{N}(\mathfrak{p})^{s}}}=\sum_{\mathfrak{a}} \frac{1}{\mathcal{N}(\mathfrak{a})^{s}}
$$

where the product runs over all prime divisors $\mathfrak{p}$ of $K$ and the sum over all integral divisors, and $\mathcal{N}(\mathfrak{a})$ is the norm of $\mathfrak{a}$. For curves over finite fields a $\zeta$-function was defined by Emil Artin in his thesis. In modified form (following F.K. Schmidt) it is given by exactly the same formula, where $K$ now stands for the function field $K / \mathbb{F}_{q}$, and $\mathcal{N}(\mathfrak{p})$ is the number of elements in the residue field, so equals $q^{\operatorname{deg} \mathfrak{p}}$. A good reference is the second volume of Hasse's collected papers, especially the paper in Italian [Has].

Example. Let $\zeta_{0}(s)$ be the $\zeta$-function of $\mathbb{P}^{1}$. The number $N(n)$ of integral divisors of degree $n$ is the number of homogeneous polynomials of degree $n$ with coefficients in $\mathbb{F}_{q}$ modulo scalars, so equals $\frac{q^{n+1}-1}{q-1}$. One finds

$$
\zeta_{0}(s)=\sum \frac{N(n)}{q^{n s}}=\frac{1}{1-\frac{q}{q^{s}}} \frac{1}{1-\frac{1}{q^{s}}}=\frac{1}{(1-q T)(1-T)},
$$

where $T=q^{-s}$.

André Weil generalised the function to schemes $X$ of finite type over $\mathbb{F}_{q}$, again by the same product formula as a product over all closed points. He conjectured that $\zeta_{X}(T)$ is a rational function of $T=q^{-s}$ (for more precise statements see [Har, Appendix C]). This was known for curves. If one sets $N_{n}=\# X\left(\mathbb{F}_{q^{n}}\right)$ then one can compute that

$$
\zeta_{X}(s)=\exp \sum_{n=1}^{\infty} \frac{N_{n}}{n} T^{n} .
$$

The number $N_{n}$ is also the the number of fixed points of the $n$th power of the Frobenius morphism $F$. The Lefschetz fixed point formula in topology computes the algebraic number $\Lambda(h)$ of fixed points (and therefore \#Fix $(h)$ if all fixed points are non-degenerate of index one) for a map $h: X \rightarrow X$ as alternating sum of traces:

$$
\Lambda(h)=\sum(-1)^{i} \operatorname{Tr}\left\{h_{*} \mid H_{i}(X, \mathbb{C})\right\} .
$$

An exercise in linear algebra gives

$$
\exp \sum_{n=1}^{\infty} \frac{\sum(-1)^{i} \operatorname{Tr}\left\{h_{*}^{n} \mid H_{i}\right\}}{n} T^{n}=\prod \operatorname{det}\left(\mathrm{Id}-T h_{*} \mid H_{i}\right)^{(-1)^{i+1}}
$$


Rationality of the $\zeta$-function follows from the existence of an appropriate cohomology theory for varieties defined over fields of finite characteristic.

Milnor then used the same formula to define $\zeta$-functions in topology [Mi1, Mi2]. Let $h: X \rightarrow X$ be a homeomorphism of a compact Euclidean Neighbourhood Retract in $\mathbb{R}^{n}$ (see [Dol, p. 81]; for this type of spaces the Lefschetz-Hopf fixed point theorem is proven in [Dol, VII.6]). Sometimes the Lefschetz number $\Lambda(h)$ can be computed as the Euler characteristic of the fixed point set of $h$. This is the case for any isometry of a compact Riemannian manifold (cf. [Mi2, Lemma 9.5]). It is true whenever the fixed point set is a deformation retract of a $h$ invariant compact neighbourhood. Let $\Lambda\left(h^{k}\right)$ be the Lefschetz number of the $k$ th iterate of $h$. Define numbers $\chi_{j}$ by the recurrence $\Lambda\left(h^{k}\right)=\sum_{j \mid k} \chi_{j}$. The number $\chi_{j}$ is the Euler characteristic of the set of points of primitive period $j$ (to have additivity of Euler characteristics also for non closed sets we use Borel-Moore homology, see [Ful, Sect. 91.1]). Then one can express the $\zeta$-function of $h$ as

$$
\zeta_{h}(T)=\prod\left(1-T^{j}\right)^{-\chi_{j} / j}
$$

We apply this to the monodromy of a function germ $f: X \rightarrow \mathbb{C}$, defined on an equidimensional space. Consider a good representative $f: \bar{X} \rightarrow D$ and let $F=f^{-1}(t)$ be the (possibly singular) Milnor fibre. In this situation one has a geometric monodromy and a $\zeta$-function $\zeta_{f}(T)$ of the monodromy. To compute the $\zeta$-function from the formula above one needs a nice model for the monodromy. In the quasi-homogeneous case it can be derived from the $\mathbb{C}^{*}$-action (cf. [Mi2, Lemma 9.4]). For a good embedded resolution one has A'Campo's construction $\left[\mathrm{A}^{\prime} \mathrm{C}\right]$.

There is a fibre bundle of pairs $\left(\bar{X}, f^{-1}(t)\right)$ over $D \backslash 0$ and a monodromy action on the relative groups $H_{*}(\bar{X}, F)$. The corresponding $\zeta$-function $\tilde{\zeta}_{f}(T)$ is related to the function $\zeta_{f}(T)$ of the monodromy action on $H_{*}(F)$ by

$$
\tilde{\zeta}_{f}(T)=(1-T)^{-1}\left(\zeta_{f}(T)\right)^{-1}
$$

It is also the inverse of the $\zeta$-function on reduced homology.

\section{Zeta and Poincaré}

The relation between Poincaré series of quasi-homogeneous singularities and $\zeta$ functions has been described by Ebeling and Gusein-Zade [EGZ].

Let $A=\oplus A_{k}$ be a graded ring with each $A_{k}$ finite dimensional. Its Poincaré series is $P_{A}(t)=\sum\left(\operatorname{dim} A_{k}\right) T^{k}$. For a general ring with a filtration the Poincaré series is by definition the Poincaré series of its associated graded ring. The Poincaré series of a singularity is the Poincaré series of its local ring.

Let $X$ be a weighted homogeneous complete intersection in $\mathbb{C}^{n}$ given by the ideal $\left(f_{1}, \ldots, f_{k}\right)$ with $\operatorname{deg} f_{i}=d_{i}$ and with wt $z_{i}=q_{i}$. Set $X^{(j)}=V\left(f_{1}, \ldots, f_{j}\right)$ for 
$j=0, \ldots, k\left(\right.$ so $X^{(0)}=\mathbb{C}^{n}$ and $\left.X^{(k)}=X\right)$. Consider the function $f_{j}$ on the space $X^{(j-1)}$ and let $F^{(j)}$ be its Milnor fibre: $F^{(j)}=f_{j}^{-1}(1) \cap X^{(j-1)}$. As monodromy transformation one can take $\left(z_{1}, \ldots, z_{k}\right) \mapsto\left(e^{2 q_{1} \pi / d_{j}} z_{1}, \ldots, e^{2 q_{n} \pi / d_{j}} z_{n}\right)$. The function $\tilde{\zeta}_{j}(T)$ is the $\zeta$-function of the monodromy action on $H^{*}\left(X^{(j-1)}, F^{(j)}\right)$. Let $Y=(X \backslash 0) / \mathbb{C}^{*}$ be the orbit space and let $Y_{m}$ be the set of orbits with isotropy group $\mathbb{Z} / m \mathbb{Z}$. One defines the orbit invariant

$$
\mathrm{Or}_{X}(T)=\prod_{m \geq 1}\left(1-T^{m}\right)^{\chi\left(Y_{m}\right)}
$$

Following Kyoji Saito [S1, S2] one defines the Saito dual (of level $d$ ) of a rational function $h(T)=\prod_{l \mid d}\left(1-T^{l}\right)^{\alpha_{l}}$ to be

$$
h^{* d}(T)=\prod_{m \mid d}\left(1-T^{d / m}\right)^{-\alpha_{m}}
$$

where the notation emphasises the dependence on the level $d$.

We can now state the result of [EGZ]:

\section{Theorem 1}

$$
P_{X}(T) \operatorname{Or}_{X}(T)=\prod_{j=1}^{k}\left(\tilde{\zeta}_{j}\right)^{*_{j}}(T)
$$

\section{Irreducible plane curve singularities}

Let $(C, 0)$ be an irreducible plane curve singularity. The $t$-adic valuation $\nu$ on $\mathbb{C}\{t\}$, the normalisation of $\mathscr{O}_{C}$, induces a filtration on $\mathscr{O}_{C}$. Let gr $\mathscr{O}_{C}$ be the associated graded algebra. Monique Lejeune-Jalabert (see Teissier's Appendix in $[$ Zar $]$ ) showed that it is a graded algebra isomorphic to $\mathbb{C}\left[C^{\Gamma}\right]=\mathbb{C}\left[t^{h}: h \in \Gamma\right]$, the ring of the monomial curve $C^{\Gamma}$ with the same semigroup $\Gamma=\left\langle\beta_{0}, \ldots, \beta_{g}\right\rangle$ as $C$.

Proposition 2 The curve $C^{\Gamma} \subset \mathbb{C}^{g+1}$ is a complete intersection.

A proof can be found in Teissier's paper. We describe the equations, using the structure of the semigroup $\Gamma=\left\langle\beta_{0}, \ldots, \beta_{g}\right\rangle$. One sets $e_{0}=\beta_{0}=n$, $e_{i}=\operatorname{gcd}\left(e_{i-1}, \beta_{i}\right), e_{i-1}=n_{i} e_{i}$. Then $\beta_{0}=n_{1} \cdots n_{g}, n_{i} \beta_{i}<\beta_{i+1}$ and $n_{i} \beta_{i} \in$ $\left\langle\beta_{0}, \ldots, \beta_{i_{1}}\right\rangle$, so $n_{i} \beta_{i}=\sum l_{i j} \beta_{j}$. Write $z_{i}=t^{\beta_{i}}$. The curve $C^{\Gamma}$ has equations

$$
f_{i}=z_{i}^{n_{i}}-\prod z_{j}^{l_{i j}}
$$

According to Teissier [loc.cit.], the curve $C$ occurs in the versal deformation of $C^{\Gamma}$ (in the part with positive weights.) In fact, each ring containing a field is a deformation of its associated graded w.r.t. any filtration. One gets also an easy way to write down equations of a plane curve with the same semigroup by taking the special deformation $\varphi_{i}=f_{i}+\lambda_{i} z_{i+1}$ with $\lambda_{i}$ a suitable power of a deformation variable $s$. The equations $\varphi_{1}, \ldots, \varphi_{g-1}$ define a smooth space on which the function $f_{g}$ gives a plane curve equisingular with the original $C$. 
Example. Let $\Gamma=\langle 4,6,13\rangle$ and set $(x, y, z)=\left(t^{4}, t^{6}, t^{13}\right)$. Equations are $y^{2}-$ $x^{3}=0$ and $z^{2}-x^{5} y=0$. We deform the first equation into $y^{2}-x^{3}=s z$. For $s \neq 0$ we can eliminate $z$ and find the plane curve $\left(y^{2}-x^{3}\right)^{2}-s^{2} x^{5} y=0$.

The result of Campillo, Delgado and Gusein-Zade [CDG] is:

Theorem 3 For an irreducible plane curve singularity the $\zeta$-function of its monodromy equals its Poincaré series.

We shall derive it from the formula of Ebeling and Gusein-Zade [EGZ] (Theorem 1). The Poincaré series in question is the same as that of $C^{\Gamma}$. The orbit invariant reduces to $\mathrm{Or}_{C^{\Gamma}}(T)=(1-T)$. To distinguish between the $\zeta$-functions occurring in the formula and monodromy $\zeta$-functions of plane curve singularities we will denote the latter by capital $\mathrm{Z}$. Theorem 3 is equivalent to the following statement:

Proposition 4 With the notations introduced above

$$
\left(\widetilde{\mathrm{Z}}_{C}(T)\right)^{-1}=\prod_{j=1}^{g}\left(\tilde{\zeta}_{j}\right)^{*_{d_{j}}}(T) .
$$

The proof will be by induction on $g$. Therefore we denote $C$ by $C_{g}$, its Milnor fibre by $F_{g}$ and $\zeta$-function by $Z_{g}(T)$. Let $C_{j}$ be the $j$ th approximate curve to $C_{g}$ (with Milnor fibre $F_{j}$ and $\zeta$-function $Z_{j}(T)$ ). It has $j$ Puiseux pairs, which equal the first $j$ Puiseux pairs of $C_{g}$. The semigroup $\Gamma_{j}$ of $C_{j}$ has $j+1$ generators. In particular, $\Gamma_{g-1}=\left\langle\beta_{0} / n_{g}, \ldots, \beta_{g-1} / n_{g}\right\rangle$. We denote the corresponding monomial curve of embedding dimension $j+1$ by $C^{(j)}$. It has the same equations as the space $X^{(j)}$, since the variables $z_{j+1}, \ldots, z_{g}$ do not occur in the first $j$ equations. Therefore $X^{(j)}=C^{(j)} \times \mathbb{C}^{g-j}$. The monodromy action on the second factor is $\left(z_{j+1}, \ldots, z_{g}\right) \mapsto\left(e^{2 q_{j+1} \pi / d_{j}} z_{j+1}, \ldots, e^{2 q_{n} \pi / d_{j}} z_{g}\right)$. The contribution to the Euler characteristic of the fixed point set, which comes from $C^{(j)} \times\left(\mathbb{C}^{g-j} \backslash 0\right)$, is zero. For the $\zeta$-function we can therefore forget about the factor. In particular, the curves $C^{(g)}$ and $C^{(g-1)}$ give rise to the same $\zeta$-functions $\tilde{\zeta}_{1}, \ldots, \tilde{\zeta}_{g-1}$. But the degrees of the equations are different: if the equation $f_{j}$ of $C^{(g)}$ has degree $d_{j}$, then it has degree $d_{j} / n_{g}$ considered as equation of $C^{(g-1)}$. In the Ebeling-GuseinZade formula (Theorem 1) the Saito dual is therefore taken at a different level. One has

$$
\left(\tilde{\zeta}_{j}\right)^{* d_{j}}(T)=\left(\tilde{\zeta}_{j}\right)^{* d_{j} / n_{g}}\left(T^{n_{g}}\right) .
$$

This said, Proposition 4 follows by induction from the following two lemmas.

Lemma $5 \quad\left(\tilde{\zeta}_{g}\right)^{*} d_{g}(T)=\left(\tilde{\zeta}_{g}(T)\right)^{-1}$.

Lemma $6 \quad \widetilde{\mathrm{Z}}_{g}(T)=\tilde{\zeta}_{g}(T) \cdot \widetilde{\mathrm{Z}}_{g-1}\left(T^{n_{g}}\right)$. 
Proof of Lemma 5. The space $X^{(g-1)}$ is homeomorphic to its normalisation, which is smooth. In coordinates $\left(\tau, z_{g}\right)$ on the normalisation the function $f_{g}$ is given by $z_{g}^{n_{g}}-\tau^{\beta_{g}}$; it has degree $d_{g}=n_{g} \beta_{g}$. One easily computes that

$$
\tilde{\zeta}_{g}(T)=\frac{\left(1-T^{\beta_{g}}\right)\left(1-T^{n_{g}}\right)}{\left(1-T^{d_{g}}\right)(1-T)}
$$

so $\tilde{\zeta}_{g}$ and its inverse are dual of level $d_{g}=n_{g} \beta_{g}$.

Proof of Lemma 6. We consider the degeneration of $C_{g}$ to the monomial curve with deformation parameter $s$. We have a complete intersection in $\left(\mathbb{C}^{g+1} \times \mathbb{C}, 0\right)$ defined by functions $\varphi_{1}(z, s), \ldots, \varphi_{g}(z, s)$. Consider the function $\Phi_{g}=\left(\varphi_{g}, s\right)$ on $\mathcal{X}^{(g-1)}=V\left(\varphi_{1}, \ldots, \varphi_{g-1}\right)$. For fixed $s \neq 0$ it defines the curve $C_{g}$ and for $s=0$ the monomial curve $C^{(g)}$. The Milnor fibre $F_{g}$ is $\Phi^{-1}(t, s)($ for $t, s \neq 0)$, while $\Phi^{-1}(t, 0)$ (for $t \neq 0$ ) gives the singular Milnor fibre $F^{(g)}$ with $n_{g}$ singular points of type $C^{(g-1)}$ (the transversal singularity of $\left.X^{(g-1)}\right)$. The intersection of a small ball around such a singular point with the Milnor fibre $F_{g}$ (for small $s$ ) is a Milnor fibre $F_{g-1}$ of the plane curve singularity $C_{g-1}$.

We are interested in the $t$-monodromy. For $\tilde{\zeta}_{g}(T)$ we have to consider the pair $\left(X^{(g-1)}, F^{(g)}\right)$. The fibre $F^{(g)}$ is a retract of $\mathcal{F}^{(g)}=\Phi^{-1}\left(t \times D_{\delta}\right)$, where $D_{\delta}$ is a small disc around the origin. In the same way we construct a thickening $\mathcal{X}^{(g-1)}$ of $X^{(g-1)}$. Then $H_{*}\left(\mathcal{X}^{(g-1)}, \mathcal{F}^{(g)}\right)=H_{*}\left(X^{(g-1)}, F^{(g)}\right)$. The Milnor fibre $F_{g}$ is now a subspace of $\mathcal{F}^{(g)}$, so it makes sense to consider the long exact sequence of the triple $\left(\mathcal{X}^{(g-1)}, \mathcal{F}^{(g)}, F_{g}\right)$ :

$$
\cdots \rightarrow H_{q}\left(\mathcal{F}^{(g)}, F_{g}\right) \longrightarrow H_{q}\left(\mathcal{X}^{(g-1)}, F_{g}\right) \longrightarrow H_{q}\left(\mathcal{X}^{(g-1)}, \mathcal{F}^{(g)}\right) \rightarrow \cdots
$$

As $\mathcal{X}^{(g-1)}$ is also contractible one has $H_{*}\left(\mathcal{X}^{(g-1)}, F_{g}\right) \cong H_{*}\left(X_{g}, F_{g}\right)$, where $X_{g}$ is the the intersection of $\mathcal{X}^{(g-1)}$ with the hyperplane $\left\{s=s_{0}\right\}$ for $s_{0}$ the same value used to define the Milnor fibre $F_{g}$, and the isomorphism is compatible with the monodromy action. Outside small neighbourhoods of the singular points of $F^{(g)}$ the thickened fibre $\mathcal{F}^{(g)}$ is a trivial fibre bundle over $D_{\delta}$ so by deformation retraction and excision $H_{*}\left(\mathcal{F}^{(g)}, F_{g}\right) \cong \bigoplus_{i=1}^{n_{g}} H^{*}\left(X_{g-1, i}, F_{g-1, i}\right)$ where $X_{g-1, i}$ is a small Milnor ball around the $i$ th singularity. The monodromy permutes the Milnor balls, and the action after $n_{g}$ steps on $H^{*}\left(X_{g-1, i}, F_{g-1, i}\right)$ is the monodromy of the curve singularity $C_{g-1}$. Therefore the $\zeta$-function of the monodromy on $\oplus_{i=1}^{n_{g}} H_{*}\left(X_{g-1, i}, F_{g-1, i}\right)$ is $\widetilde{\mathrm{Z}}_{g-1}\left(T^{n_{g}}\right)$. The lemma now follows from the exact sequence

$$
\cdots \rightarrow \oplus_{i=1}^{n_{g}} H_{q}\left(X_{g-1, i}, F_{g-1, i}\right) \longrightarrow H_{q}\left(X_{g}, F_{g}\right) \longrightarrow H_{q}\left(X^{(g-1)}, F^{(g)}\right) \rightarrow \cdots
$$




\section{References}

[A'C] Norbert A'Campo, La function zêta d'une monodromie. Comment. Math. Helvetici 50 (1975), 233-248.

[CDG] S.M. Gusein-Zade, F. Delgado and A. Campillo, On the monodromy of a plane curve singularity and the Poincaré series of its ring of functions. Funct. Anal. Appl. 33 (1999), 56-57 (translation from Funkts. Anal. Prilozh. 33:1 (1999) $66-68)$.

[Dol] Albrecht Dold, Lectures on Algebraic Topology. Berlin etc., Springer 1972 (Grundlehren der mathematischen Wissenschaften; 200).

[Eb] Wolfgang Ebeling, Poincaré series and monodromy of a two-dimensional quasihomogeneous hypersurface singularity. Manuscripta Math. 107 (2002), 271-282.

[EGZ] W. Ebeling and S.M. Gusein-Zade, Monodromies and Poincaré series of quasihomogeneous complete intersections. Preprint, 2003.

[Ful] William Fulton, Intersection Theory. Berlin etc., Springer 1984 (Ergebnisse der Mathematik; 3. Folge, 2).

[Har] Robin Hartshorne, Algebraic Geometry. New York etc., Springer 1977 (Graduate texts in Math; 52).

[Has] Helmut Hasse, Punti razionali sopra curve algebriche a congruenze. In: Reale Accademia d'Italia, Fondazione Alessandro Volta, Atti dei Convegni 9, pp. 85140, Roma (1943). Also in: Mathematische Abhandlungen. Band 2. Walter de Gruyter, Berlin-New York, 1975, pp. 295-350.

[Mi1] John Milnor, Infinite cyclic coverings. In: Conference on the Topology of Manifolds, Prindle, Weber \& Schmidt, Boston, 1968, pp. 115-133.

[Mi2] John Milnor, Singular points of complex hypersurfaces. Princeton University Press 1968 (Annals of Mathematics Studies; 61).

[S1] Kyoji Saito, Duality for Regular Systems of Weights: a précis. In: Topological Field Theory, Primitive Forms, and Related Topics. (Progr. Math. 160) Birkhäuser, Boston (1998) pp. 379-426.

[S2] Kyoji Saito, Duality for Regular Systems of Weights. Asian J. Math. 2 (1998), 983-1048.

[Zar] Oscar Zariski, Le problème des modules pour les branches planes. Cours donné au Centre de Mathématiques de l'École Polytechnique. Nouvelle éd. revue par l'auteur. Rédigé par François Kmety et Michel Merle. Avec un appendice de Bernard Teissier. Hermann, Éditeurs des Sciences et des Arts, Paris, 1986.

Matematik

Chalmers tekniska högskola och Göteborgs universitet,

SE 41296 Göteborg, Sweden

e-mail: stevens@math. chalmers.se 